\title{
Holographic superconductors in a rotating spacetime
}

\author{
Kai Lin ${ }^{\mathrm{a}}$, E. Abdalla ${ }^{\mathrm{b}}$ \\ Instituto de Física, Universidade de São Paulo, CP 66318, São Paulo 05315-970, Brazil
}

Received: 8 September 2014 / Accepted: 20 October 2014 / Published online: 5 November 2014

(c) The Author(s) 2014. This article is published with open access at Springerlink.com

\begin{abstract}
We consider holographic superconductors in a rotating black string spacetime. In view of the mandatory introduction of the $A_{\varphi}$ component of the vector potential we are left with three equations to be solved. Their solutions show that the rotation parameter $a$ influences the critical temperature $T_{\mathrm{c}}$ and the conductivity $\sigma$ in a simple but non-trivial way.
\end{abstract}

\section{Introduction}

The use of the AdS/CFT (Anti-de Sitter/Conformal Field Theory) correspondence has been recently proposed by Hartnoll, Herzog, and Horowitz [1,2] for the investigation of the strongly correlated condensed matter physics from the gravitational dual. It generally shows that the instability of black strings corresponds to a second-order phase transition from the normal state to a superconductor state with the spontaneous $U(1)$ symmetry breaking and leads to interesting physics in the related lower-dimensional physics.

Such a breakthrough result has been widely used more recently to model conductivity and other condensed matter physics properties, in particular, holographic superconductors in various spacetimes [3-22]. Most authors considered, up to now, the holographic superconductor on a static background. However, we still have room for the other hair, namely angular momentum, on top of mass and charge. Or, equivalently, we can say that a real black string (or black hole) could have angular momentum in the background. It is thus our goal to study the holographic superconductor related to the 4-dimensional rotating black hole. We consider the simplest uncharged background case and we will use both analytical and numerical methods to find the critical temperature and conductivity of the holographic superconductor related to the rotating black hole background.

\footnotetext{
a e-mail: 1k314159@hotmail.com

be-mail: eabdalla@usp.br
}

The paper is planned as follows. In Sect. 2, we introduce the metric of the uncharged rotating black hole and calculate the Hawking radiation and Hawking temperature. Then the analytical and numerical methods are applied to find the conductivity of such a black hole in Sects. 3 and 4, respectively. In Sects. 5 and 6, we want to improve the analytical method to research the holographic superconductor with backreactions. Section 7 includes a summary and a conclusion.

\section{Uncharged rotating black strings and Hawking temperature}

The simplest uncharged rotating black string in 4-dimensional ADS spacetime is given by [24-26]

$$
\begin{aligned}
\mathrm{d} s^{2}= & -f(r)(\Xi \mathrm{d} t-a \mathrm{~d} \varphi)^{2}+r^{2}\left(\frac{a}{l^{2}} \mathrm{~d} t-\Xi \mathrm{d} \varphi\right)^{2} \\
& +\frac{\mathrm{d} r^{2}}{f(r)}+\frac{r^{2}}{l^{2}} \mathrm{~d} \theta^{2},
\end{aligned}
$$

where $f(r)=\frac{r^{2}}{l^{2}}-\frac{2 M}{r}$ and $\Xi=1+\frac{a^{2}}{l^{2}} ; M$ and $a$ are the mass and rotation parameters of the black string, while $\Lambda=-3 / l^{2}$ is the cosmological constant. Without loss of generality, we set $l=1$ and rewrite the function $f(r)$ as

$f(r)=r^{2}-\frac{r_{h}^{3}}{r}$,

where $r_{h}$ is the event horizon of the AdS black hole. Although a very simple model of rotation, the results will show a dependence on the above rotation parameter $a$. Rotation is one of the few possible hairs of a black hole and corresponds to a general solution of the end fate of a collapsing star. The solution we are considering is simpler than the full rotating Kerr solution, but it is a sensible and consistent model. On the other hand, rotation in a physical condensed matter system is generally related to spin and corresponds also to a generic physical system. 
We first find the Hawking radiation and temperature of such a black hole solution. A simple and effective method to study the Hawking radiation is the tunneling radiation theory of black holes proposed by Robinson and Wilczek et al. [27-29]. Developing the tunneling theory, the HamiltonJacobi method has been proposed to compute the Hawking tunneling rate and Hawking radiation at the horizon of the black hole [30-34]. We use that method to find the Hawking tunneling radiation and the temperature of the black hole.

Let us consider the semi-classical Hamilton-Jacobi equation [30-34]

$g^{\mu \nu} \frac{\partial S}{\partial x^{\mu}} \frac{\partial S}{\partial x^{\nu}}+\mu_{0}^{2}=0$,

where $\mu_{0}$ is the mass of the radiation particle. We shall use a semi-classical approximation to find the Hawking radiation and temperature of the black hole.

In the rotating black string spacetime, we separate the Hamilton-Jacobi equation as

$S=-\omega_{0} t+R(r)+m_{0} \varphi+Y(\theta)$,

where $\omega_{0}$ and $m_{0}$ are the energy and angular momentum of particles, respectively, so that we obtain the radial and angular Hamilton-Jacobi equations as

$$
\begin{aligned}
& f^{2} R^{\prime 2}-\frac{\left(\Xi \omega_{0}-m_{0} a\right)^{2}}{\left(a^{2}-\Xi^{2}\right)^{2}} \\
& +f\left[\frac{\left(a \omega_{0}-m_{0} \Xi\right)^{2}}{r^{2}\left(a^{2}-\Xi^{2}\right)^{2}}+\frac{\lambda}{r^{2}}+\mu_{0}^{2}\right]=0, \\
& \left(\frac{\mathrm{d} Y}{\mathrm{~d} \theta}\right)^{2}=\lambda,
\end{aligned}
$$

where $\lambda$ is a constant. At the event horizon $r_{0}$, we can expand the function $f$ as

$f(r)=f^{\prime}\left(r_{h}\right)\left(r-r_{h}\right)+\frac{f^{\prime \prime}\left(r_{h}\right)}{2}\left(r-r_{h}\right)^{2}+\cdots$,

and we get

$R_{ \pm}= \pm \frac{i \pi}{f^{\prime}\left(r_{h}\right)} \frac{\omega_{0}-\frac{m_{0} a}{\Xi}}{\Xi-\frac{a^{2}}{\Xi}}$

where $R_{+}$and $R_{-}$are the radial outgoing and incoming modes, respectively [27-29]. Therefore, the tunneling rate at the event horizon is

$\Gamma=e^{-2\left(\Im R_{+}-\Im R_{-}\right)}=\exp \left(-\frac{4 \pi}{f^{\prime}\left(r_{h}\right)} \frac{\omega_{0}-\frac{m_{0} a}{\Xi}}{\Xi-\frac{a^{2}}{\Xi}}\right)$,

while the Hawking temperature is given by [27-29]

$T_{h}=\frac{f^{\prime}\left(r_{h}\right)}{4 \varrho \pi}=\frac{3 r_{h}}{4 \varrho \pi}$

where $\varrho=\frac{\Xi}{\Xi^{2}-a^{2}}=\frac{1+a^{2}}{1+a^{2}+a^{4}}$.
We thus find that the form of the Hawking temperature is very similar to the temperature of static black hole, while the $\varrho$ in Eq. (2.9) depends on the rotation parameter $a$. We use the results to analyze the holographic superconductor in the next section.

\section{Holographic superconductor modes and analytical investigation}

In the rotating black hole spacetime background, the Lagrangian density of the simplest holographic superconductor model with a Maxwell field and a charged complex scalar field is

$\mathcal{L}=-\frac{1}{4} F^{\mu \nu} F_{\mu \nu}-|\partial \Psi-i A \Psi|^{2}-\frac{m^{2}}{L^{2}} \Psi^{2}$,

where $F_{\mu \nu}=\partial_{\mu} A_{\nu}-\partial_{\nu} A_{\mu}$ and $\Psi=\Psi(r)$ since $g_{\mu \nu}$ only depends on $r$. As in the static background spacetime case, $A_{\mu}=\delta_{\mu}^{t} \Phi(r)$, but in the rotating black string background, we must set $A_{\mu}=\delta_{\mu}^{t} \Phi(r)+\delta_{\mu}^{\varphi} \Omega(r)$ in view of the presence of the $g_{t \varphi}$ term.

From the variation of Eq. (3.1), we get three equations. They are

$$
\begin{aligned}
& \Psi^{\prime \prime}+\left(\frac{f^{\prime}}{f}+\frac{2}{r}\right) \Psi^{\prime}+\left[\frac{\left(1+a^{2}\right) \Phi+a \Omega}{\left(1+a^{2}+a^{4}\right) f}\right]^{2} \Psi \\
& +\left[\frac{\left(1+a^{2}\right) \Omega+a \Phi}{r\left(1+a^{2}+a^{4}\right) f}\right]^{2} \Psi-\frac{m^{2}}{L^{2} f} \Psi=0, \\
& \Phi^{\prime \prime}+\left[\frac{2\left(1+a^{2}\right)^{2}}{\left(1+a^{2}+a^{4}\right) r}-\frac{a^{2} f^{\prime}}{\left(1+a^{2}+a^{4}\right) f}\right] \Phi^{\prime} \\
& -\frac{2 \Psi^{2}}{f} \Phi+\left[\frac{2\left(a+a^{3}\right)}{\left(1+a^{2}+a^{4}\right) r}-\frac{a\left(1+a^{2}\right) f^{\prime}}{\left(1+a^{2}+a^{4}\right) f}\right] \Omega^{\prime}=0, \\
& \Omega^{\prime \prime}-\left[\frac{2 a^{2}}{\left(1+a^{2}+a^{4}\right) r}-\frac{\left(1+a^{2}\right)^{2} f^{\prime}}{\left(1+a^{2}+a^{4}\right) f}\right] \Omega^{\prime} \\
& -\frac{2 \Psi^{2}}{f} \Omega-\left[\frac{2\left(a+a^{3}\right)}{\left(1+a^{2}+a^{4}\right) r}-\frac{a\left(1+a^{2}\right) f^{\prime}}{\left(1+a^{2}+a^{4}\right) f}\right] \Phi^{\prime}=0 .
\end{aligned}
$$

The boundary condition at the horizon requires $A_{\mu}$ to be finite. The solutions of the above equations at infinity are given by

$$
\begin{aligned}
\Psi & =\frac{\sqrt{2}\left\langle\mathcal{O}_{1}\right\rangle}{r^{\Delta_{-}}}+\frac{\sqrt{2}\left\langle\mathcal{O}_{2}\right\rangle}{r^{\Delta_{+}}}+\cdots, \\
\Phi & =\mu-\frac{\rho}{r}+\cdots, \\
\Omega & =v-\frac{\zeta}{r}+\cdots,
\end{aligned}
$$


where $\Delta_{ \pm}=\frac{3}{2} \pm \sqrt{\frac{9}{4}+\frac{m^{2}}{L^{2}}}$ implying that $\Delta$ satisfies $\frac{1}{2}<$ $\Delta<3$. In the following, we set $L=1$.

Siopsis and Therrien [35] proposed an effective analytic method to calculate the critical temperature of holographic superconductor in static spacetime. We generalize such a method for the rotating black hole case in this section.

According to that procedure, we change the coordinates as $z=r_{0} / r$ and the field equations can be rewritten as

$$
\begin{aligned}
& z \Psi_{, z z}-\frac{2+z^{3}}{1-z^{3}} \Psi_{, z}+\frac{z\left[\left(a^{2}+1\right) \Phi+a \Omega\right]^{2}}{\left(a^{4}+a^{2}+1\right)^{2} r_{0}^{2}\left(1-z^{3}\right)^{2}} \Psi \\
& +\frac{z\left(\left(a^{2}+1\right) \Omega+a \Phi\right)^{2}}{\left(a^{4}+a^{2}+1\right)^{2} r_{0}^{2}\left(z^{3}-1\right)} \Psi-\frac{m^{2}}{z\left(1-z^{3}\right)} \Psi=0, \\
& \Phi_{, z z}-\frac{3 a^{2} z^{2} \Phi_{, z}}{\left(a^{4}+a^{2}+1\right)\left(z^{3}-1\right)}-\frac{2 \Psi^{2}}{z^{2}\left(1-z^{3}\right)} \Phi \\
& -\frac{3 a\left(a^{2}+1\right) z^{2} \Omega_{, z}}{\left(a^{4}+a^{2}+1\right)\left(z^{3}-1\right)}=0, \\
& \Omega_{, z z}+\frac{3\left(a^{2}+1\right)^{2} z^{2} \Omega, z}{\left(a^{4}+a^{2}+1\right)\left(z^{3}-1\right)}-\frac{2 \Psi^{2}}{z^{2}\left(1-z^{3}\right)} \Omega \\
& +\frac{3 a\left(a^{2}+1\right) z^{2} \Phi_{, z}}{\left(a^{4}+a^{2}+1\right)\left(z^{3}-1\right)}=0 .
\end{aligned}
$$

At the critical temperature $T_{\mathrm{c}}$, we have $\Psi=0$, thus Eqs. (3.7) and (3.8) become

$$
\begin{aligned}
\Phi_{, z z}- & \frac{3 a^{2} z^{2} \Phi_{, z}}{\left(a^{4}+a^{2}+1\right)\left(z^{3}-1\right)} \\
- & \frac{3 a\left(a^{2}+1\right) z^{2} \Omega_{, z}}{\left(a^{4}+a^{2}+1\right)\left(z^{3}-1\right)}=0 \\
\Omega_{, z z}+ & \frac{3\left(a^{2}+1\right)^{2} z^{2} \Omega_{, z}}{\left(a^{4}+a^{2}+1\right)\left(z^{3}-1\right)} \\
& +\frac{3 a\left(a^{2}+1\right) z^{2} \Phi_{, z}}{\left(a^{4}+a^{2}+1\right)\left(z^{3}-1\right)}=0 .
\end{aligned}
$$

Therefore, we can rewrite the above equations as $\Omega_{, z}=$ $\Omega_{, z}\left(\Phi_{, z z}, \Phi_{, z}\right)$ and $\Phi_{, z}=\Phi_{, z}\left(\Omega_{, z z}, \Omega_{, z}\right)$, and then substitute into Eq. (3.9) again, so that the decoupling equations are given by

$$
\begin{aligned}
& \Phi_{, z z z}+\frac{2}{z} \frac{2 z^{3}+1}{z^{3}-1} \Phi_{, z z}=0 \\
& \Omega_{, z z z}+\frac{2}{z} \frac{2 z^{3}+1}{z^{3}-1} \Omega_{, z z}=0
\end{aligned}
$$

and the solutions are

$$
\begin{aligned}
\Phi= & \mu-\rho z+C_{1}\left[\sqrt{12} \arctan \left(\frac{1+2 z}{\sqrt{3}}\right)\right. \\
& \left.+\ln \left(\frac{1+z+z^{2}}{(1-z)^{2}}\right)\right]
\end{aligned}
$$

$$
\begin{aligned}
\Omega= & v-\zeta z+C_{2}\left[\sqrt{12} \arctan \left(\frac{1+2 z}{\sqrt{3}}\right)\right. \\
& \left.+\ln \left(\frac{1+z+z^{2}}{(1-z)^{2}}\right)\right] .
\end{aligned}
$$

Considering the boundary condition at the horizon, we require $\left.\Phi\right|_{z=1}=\left.\Omega\right|_{z=1}=0$, so that we may set

$$
\begin{aligned}
& \Phi=\lambda r_{h c}(1-z), \\
& \Omega=\bar{\lambda} r_{h c}(1-z),
\end{aligned}
$$

where $\lambda=\frac{\rho}{r_{h c}^{2}}$ [35], and $r_{h c}$ is the radius of the horizon at critical temperature. Next, substituting Eq. (3.12) into Eq. (3.9), we find $\bar{\lambda}=-\frac{a}{1+a^{2}} \lambda$.

According to the idea of Siopsis and Terrien, we introduce $\Psi(z)=\frac{\left\langle\mathcal{O}_{\Delta}\right\rangle}{\sqrt{2} r_{h}^{\Delta}} z^{\Delta} F(z)\left(\right.$ where $\left.F\right|_{z=0}=1$, and $\left.F_{, z}\right|_{z=0}=0$ ) to match the boundary condition, and substitute Eq. (3.12) into Eq. (3.6), which becomes

$$
\begin{aligned}
& -F_{, z z}+\frac{1}{z}\left(\frac{2+z^{3}}{1-z^{3}}-2 \Delta\right) F_{, z}+\frac{\Delta^{2} z}{1-z^{3}} F \\
& =\frac{\tilde{\lambda}^{2}}{\left(1+z+z^{2}\right)^{2}} F,
\end{aligned}
$$

where $\tilde{\lambda}=\frac{\lambda}{1+a^{2}}$. We observe that the form of Eq. (3.13) is the same as the result of [35], so we can directly use the mathematical conclusions about the eigenvalue $\tilde{\lambda}$,

$\tilde{\lambda}^{2}=\frac{\int_{0}^{1} \mathrm{dz}\left\{z^{2 \Delta-2}\left[\left(1-z^{3}\right)\left(F_{, z}\right)^{2}+\Delta^{2} z F^{2}\right]\right\}}{\int_{0}^{1} \mathrm{~d} z\left[z^{2 \Delta-2} \frac{1-z}{1+z+z^{2}} F^{2}\right]}$,

and we assume a very simple trial function $F=F_{\alpha}(z) \equiv$ $1-\alpha z^{2}$. We can thus compute the minimizing value of $\tilde{\lambda}^{2}$. Then, using the temperature's formula, the critical temperature is given by the expression

$T_{\mathrm{c}}=\frac{3}{4 \varrho \pi} r_{h c}=\frac{3}{4 \varrho \pi} \sqrt{\frac{\rho}{\lambda}}=\frac{3}{4 \pi} \eta \sqrt{\frac{\rho}{\tilde{\lambda}}}$,

where

$\eta=\frac{1}{\varrho \sqrt{1+a^{2}}}=\frac{1+a^{2}+a^{4}}{\left(1+a^{2}\right)^{3 / 2}}$.

From the above equations, we can get the relation between the critical temperature $T_{\mathrm{c}}$ and $\tilde{\lambda}$, which depends on $\Delta$.

In Table 1 we write the results of the analytical method in the rotating black strings spacetime.

It is clearly evident that the conclusion is no other than the results of [35] as $\eta=1(a=0)$, and all the corrections depend on $\eta$; thus we focus on $\eta$.

In Fig. 1 we plot the relationship between $\eta$ and $a$. We find that $\eta<1$ as $|a|<a_{1}$ where $a_{1}$ $=\sqrt{\frac{2^{2 / 3}}{6}\left[(25+3 \sqrt{69})^{1 / 3}+(25-3 \sqrt{69})^{1 / 3}\right]-\frac{1}{3}} \approx 0.868837$. In this region, the correction from $a$ led the critical temperature $T_{\mathrm{c}}$ to decrease. The minimum value of $\eta$ is $\eta_{\min }=$ 

temperature $T_{\mathrm{c}}$ and $\Delta$
Table 1 Relation of the critical

\begin{tabular}{ll}
\hline$\Delta$ & $T_{\mathrm{c}}$ \\
\hline 0.6 & $0.45504 \eta \sqrt{\rho}$ \\
0.8 & $0.29124 \eta \sqrt{\rho}$ \\
1 & $0.22496 \eta \sqrt{\rho}$ \\
1.2 & $0.18638 \eta \sqrt{\rho}$ \\
1.4 & $0.16066 \eta \sqrt{\rho}$ \\
1.6 & $0.14214 \eta \sqrt{\rho}$ \\
1.8 & $0.12810 \eta \sqrt{\rho}$ \\
2 & $0.11704 \eta \sqrt{\rho}$ \\
2.2 & $0.10809 \eta \sqrt{\rho}$ \\
2.4 & $0.10067 \eta \sqrt{\rho}$ \\
2.6 & $0.09440 \eta \sqrt{\rho}$ \\
2.8 & $0.08903 \eta \sqrt{\rho}$ \\
\hline
\end{tabular}

$\sqrt{\frac{26 \sqrt{13}}{27}-\frac{70}{27}} \approx 0.937774$ at $a_{\min }=\sqrt{\frac{\sqrt{13}-3}{2}} \approx 0.550251$. However, the effect of $\eta$ leads to an increase of the critical temperature $T_{\mathrm{c}}$ as $|a|>a_{1}$.

Further details of holographic superconductor shall be investigated by a numerical method in the next section.

\section{Numerical investigation and conductivity}

In this section, we set $m^{2}=-2, r_{h}=1$, and then use the shooting method [1,2], combining with the boundary condition (3.5) and the main equations (3.2)-(3.4), to calculate the condensate as a function of temperature. The results are shown in Fig. 2.

In Fig. 2, it is easy to find that, as the rotation parameter $a$ increases, the curved line gets lower.

Finally, we study the conductivity. Considering the perturbed Maxwell field $\mathrm{d} A=A_{x}(r) e^{-i \omega t} \mathrm{~d} x$, we obtain the equation

$$
\begin{aligned}
& A_{x}^{\prime \prime}+\frac{f^{\prime}}{f} A_{x}^{\prime}-\varrho^{2}\left[1-\frac{f a^{2}}{\left(1+a^{2}\right)^{2} r^{2}}\right] \frac{\omega^{2}}{f^{2}} A_{x} \\
& -2 \frac{\Psi^{2}}{f} A_{x}=0 .
\end{aligned}
$$

The boundary condition at the event horizon requires

$$
A_{x}(r) \sim f(r)^{-i \varrho \omega / 3 r_{h}}
$$

while the behavior of $A_{x}$ in the asymptotic AdS region is

$$
A_{x}=A^{(0)}+\frac{A^{(1)}}{r} \text {. }
$$

Therefore, we can get the conductivity of the superconductor by using the AdS/CFT dictionary $[1,2]$

$\sigma=-\frac{i A^{(1)}}{\omega A^{(0)}}$.

We use the above equations to calculate the conductivity in Figs. 3 and 4. It can be seen that the effect of $a$ drives the real part of the conductivity to smaller and smaller values.
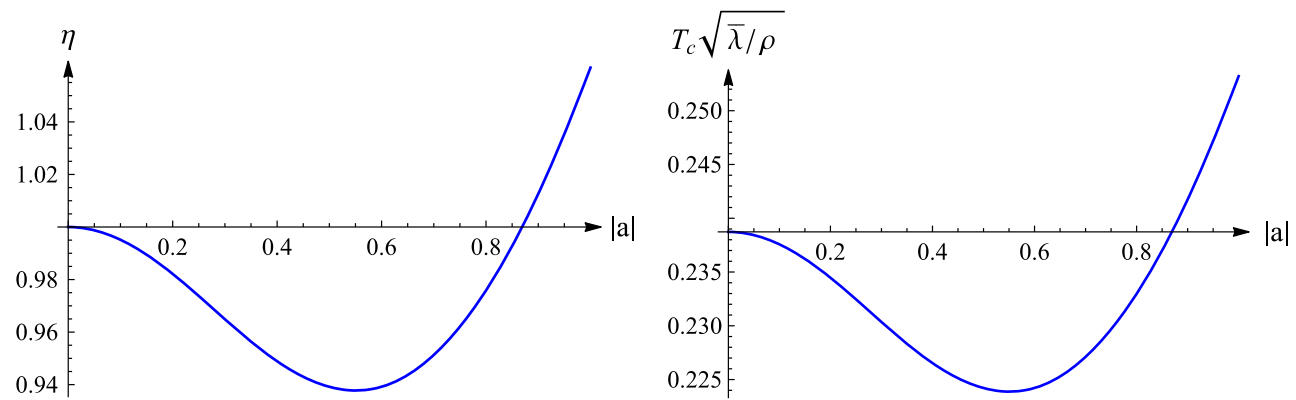

Fig. 1 The relationship between $\eta$ and $|a|$ according to Eq. (3.16) and in the second diagram the dependence of the temperature with $|a|$
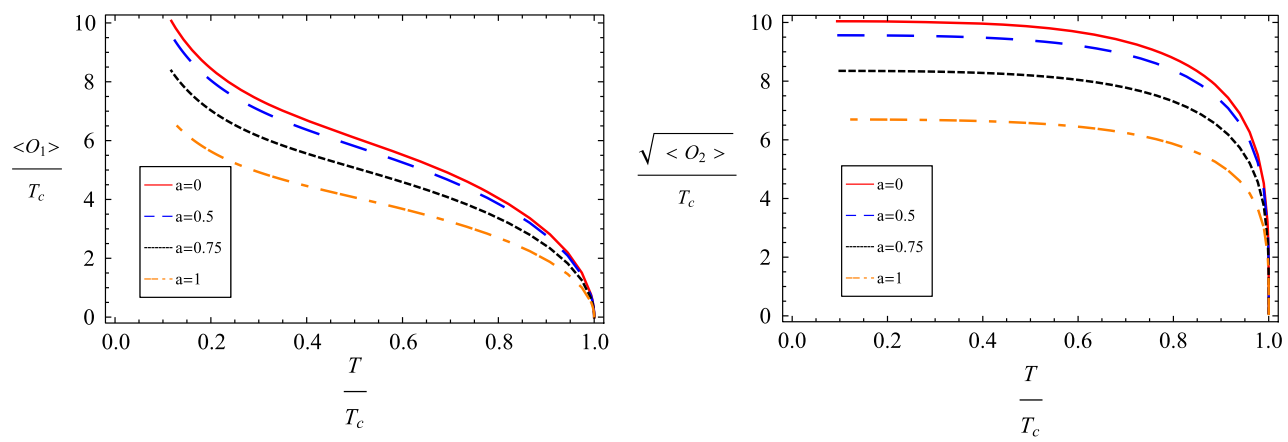

Fig. 2 The condensate as a function of the temperature for the two operators $\mathcal{O}_{1}$ and $\mathcal{O}_{2}$ in rotating spacetime 

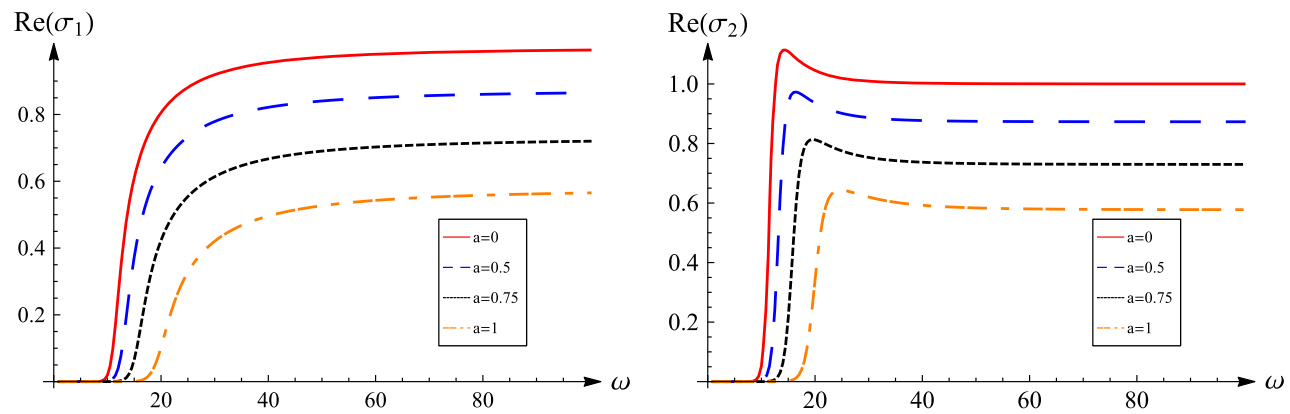

Fig. 3 The real part of conductivity for the two operators $\mathcal{O}_{1}$ and $\mathcal{O}_{2}$ in rotating spacetime
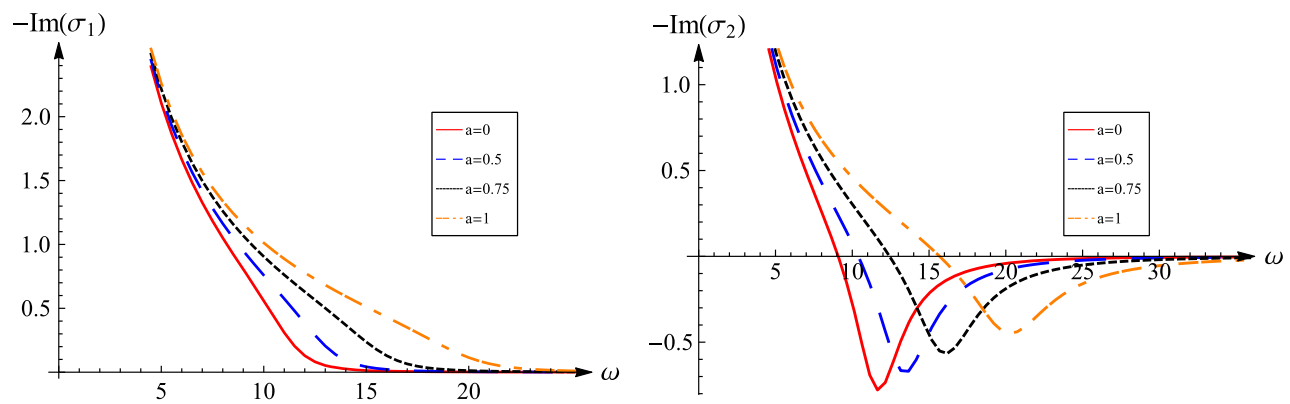

Fig. 4 The imaginary part of conductivity for the two operators $\mathcal{O}_{1}$ and $\mathcal{O}_{2}$ in rotating spacetime
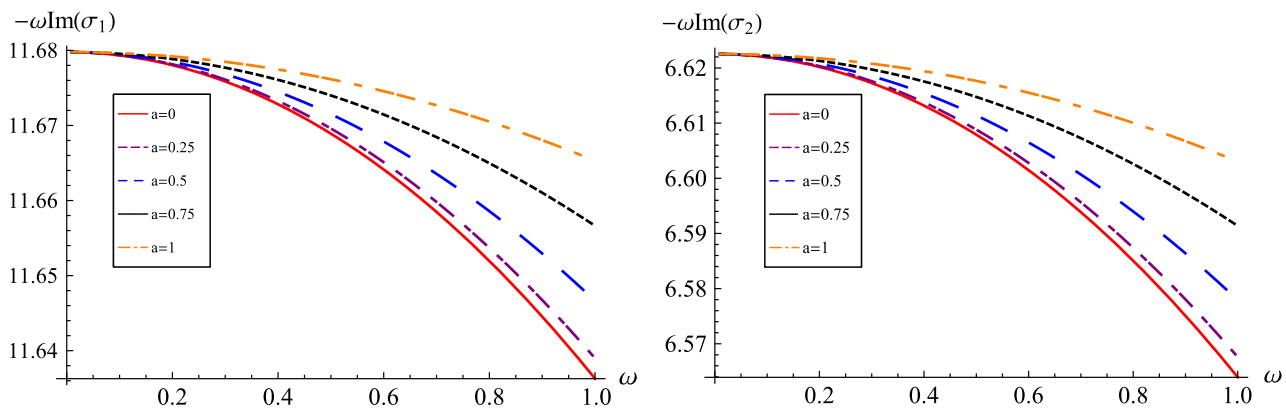

Fig. $5-\omega \operatorname{Im} \sigma$ for the two operators $\mathcal{O}_{1}$ and $\mathcal{O}_{2}$ in rotating spacetime

For the imaginary part the behavior is more involved: the frequency times the imaginary part increases in absolute value, as we see in Fig. 5, where we plot $-\omega \operatorname{Im} \sigma$ with small $\omega$. The results also show that $-\omega \operatorname{Im} \sigma$ with different $a$ go to the same constant as $\omega$ goes to 0 . This implies superconductivity for zero frequency.

\section{Modified analytical method with backreaction}

The authors of reference [36] proposed a method to analytically research the holographic superconductor with backreaction. However, using such a method still poses a problem: the error will be larger as the backreaction increases. Here, we improve the method in [36] to get more precise results and use the improved method to study the holographic superconductors with backreaction in a rotating black strings spacetime.
Let us consider the general action of holographic superconductor model in the $d$-dimensional spacetime,

$$
\begin{aligned}
S= & \int \mathrm{d}^{d} x \sqrt{-g}\left[\frac{1}{2 \kappa^{2}}(R-2 \Lambda)-\frac{1}{4} F^{\mu \nu} F_{\mu \nu}\right. \\
& \left.-|\partial \psi-i A \psi|^{2}-m^{2} \psi^{2}\right]
\end{aligned}
$$

where $\kappa^{2}=8 \pi G_{d}, \Lambda=-\frac{1}{2 L^{2}}(d-1)(d-2), \mathrm{d} A=\phi(r) \mathrm{d} t$, and $\psi(r)$ are the $d$-dimensional gravitational constant, the cosmological constant, the gauge field, and the scalar field, respectively.

In this section, we use $d$-dimensional static spacetime as an example to show the improved analytical method of holographic superconductor. In [36], the authors focus on the $d$-dimensional planar Anti-de Sitter black hole 
$\mathrm{d} s^{2}=-f(r) e^{-\chi(r)} \mathrm{d} t^{2}+\frac{\mathrm{d} r^{2}}{f(r)}+r^{2} h_{i j} \mathrm{~d} x^{i} \mathrm{~d} x^{j}$,

where $h_{i j} \mathrm{~d} x^{i} \mathrm{~d} x^{j}$ is the line element of the $(d-2)$ dimensional hypersurface with the curvature $k=0$.

Considering the backreaction from thespacetime, we fail to directly get the results of $f(r)$ and $\chi(r)$, while the effect from $\psi$ is very small, so that we can expand them as

$$
\begin{aligned}
& f=f_{0}+\epsilon^{2} f_{2}+\epsilon^{4} f_{4}+\cdots \\
& \chi=\chi_{0}+\epsilon^{2} \chi_{2}+\epsilon^{4} \chi_{4}+\cdots,
\end{aligned}
$$

where $\epsilon \ll 1$. According to the calculation in [36], at the critical temperature,

$\phi_{c}(z)=\lambda r_{c}\left(1-z^{d-3}\right)$,

where $\phi_{c}$ and $r_{c}$ are $A_{t}=\phi$ and event horizon position at the critical temperature, while $z=\frac{r_{c}}{r}$ and $\lambda=\rho / r_{c}^{d-2}$ ( $\rho$ is the charge density). Therefore, we can get $f_{0}$ and $\chi_{0}$,

$$
\begin{aligned}
f_{0} & =r_{c}^{2} g(z) \\
& =r_{c}^{2}\left[\frac{1}{L^{2} z^{2}}-\frac{z^{d-3}}{L^{2}}-\kappa^{2} \lambda^{2} \frac{d-3}{d-2} z^{d-3}\left(1-z^{d-3}\right)\right], \\
\chi_{0} & =0 .
\end{aligned}
$$

At first order, we can set $\psi=\frac{\psi_{C}}{r^{\Delta}} z^{\Delta} F(z)$ (where $m^{2}=$ $\Delta(1-d+\Delta)$ and $\psi_{0}$ is a constant $)$ and the asymptotic Antide Sitter boundary condition for $\psi$ requires $F(0)=1$ and $F^{\prime}(0)=0$. Finally, the equation of motion for $F(z)$ in [36] is given by

$$
\begin{aligned}
& F^{\prime \prime}+\left[\frac{2(\Delta+2)-d}{z}+\frac{g^{\prime}}{g}\right] F^{\prime}+\left[\lambda^{2} \frac{\left(1-z^{d-3}\right)^{2}}{z^{4} g^{2}}\right. \\
& \left.+\frac{\Delta}{z}\left(\frac{\Delta+3-d}{z}+\frac{g^{\prime}}{g}\right)-\frac{m^{2}}{z^{4} g}\right] F=0 .
\end{aligned}
$$

From the Sturm-Liouville eigenvalue problem, we get

$$
\lambda^{2}=\frac{\int_{0}^{1} T\left(F^{\prime 2}-U F^{2}\right) \mathrm{d} z}{\int_{0}^{1} T V F^{2} \mathrm{~d} z},
$$

where

$$
\begin{aligned}
T & =z^{2(\Delta+2)-d} g, \\
U & =\frac{\Delta}{z}\left(\frac{\Delta+3-d}{z}+\frac{g^{\prime}}{g}\right)-\frac{m^{2}}{z^{4} g}, \\
V & =\frac{\left(1-z^{d-3}\right)^{2}}{z^{4} g^{2}},
\end{aligned}
$$

and we assume $F(z)=1-a z^{2}$, which satisfies the AdS boundary condition.

At the probe limit, we can calculate $\lambda^{2}$ at the critical temperature as the minimum eigenvalue in Eq. (5.8) andcompute
Table 2 Comparing the $T_{\mathrm{c}}$ results of the iteration method and the method of [36] with $\Delta \kappa=0.025$ in the 5-dimensional AdS black hole background, where $m^{2} L^{2}=-3$

\begin{tabular}{llll}
\hline$\kappa$ & Iteration method & Method of [36] & Numerical \\
\hline 0 & $0.1962 \rho^{1 / 3}$ & $0.1962 \rho^{1 / 3}$ & $0.1980 \rho^{1 / 3}$ \\
0.05 & $0.1934 \rho^{1 / 3}$ & $0.1934 \rho^{1 / 3}$ & $0.1953 \rho^{1 / 3}$ \\
0.10 & $0.1854 \rho^{1 / 3}$ & $0.1853 \rho^{1 / 3}$ & $0.1874 \rho^{1 / 3}$ \\
0.15 & $0.1726 \rho^{1 / 3}$ & $0.1722 \rho^{1 / 3}$ & $0.1748 \rho^{1 / 3}$ \\
0.20 & $0.1558 \rho^{1 / 3}$ & $0.1549 \rho^{1 / 3}$ & $0.1580 \rho^{1 / 3}$ \\
0.25 & $0.1361 \rho^{1 / 3}$ & $0.1345 \rho^{1 / 3}$ & $0.1382 \rho^{1 / 3}$ \\
0.30 & $0.1147 \rho^{1 / 3}$ & $0.1123 \rho^{1 / 3}$ & $0.1165 \rho^{1 / 3}$ \\
\hline
\end{tabular}

the critical temperature $T_{\mathrm{c}}$ by the relationship between $\lambda$ and $T_{\mathrm{c}}$.

However, when considering the backreaction from spacetime, we must face the troubling fact that $g$ in Eq. (5.5) includes $\lambda^{2}$ as $\kappa \neq 0$. In order to solve this difficulty, the authors in [36] express $\kappa$ as

$\kappa_{n}=n \Delta \kappa, \quad n=0,1,2 \ldots$,

where $\Delta \kappa=\kappa_{n+1}-\kappa_{n}$ and $\kappa_{0}=0$, so we can use the $\left.\lambda^{2}\right|_{\kappa_{n-1}}$ to replace $\lambda^{2}$ in $g(r)$, which can be rewritten as

$g \approx \frac{1}{L^{2} z^{2}}-\frac{z^{d-3}}{L^{2}}-\kappa_{n}^{2}\left(\left.\lambda^{2}\right|_{\kappa_{n-1}}\right) \frac{d-3}{d-2} z^{d-3}\left(1-z^{d-3}\right)$.

The authors of [36] got good results by using the above method, but the error between exact numerical values and the values from the above method are larger as $\kappa$ increases.

We want to use the iteration method to improve the above method, so let us consider the $\left.\lambda^{2}\right|_{\kappa_{n-1}}$ (or directly $\left.\lambda^{2}\right|_{\kappa_{0}}$ ) as the initial value of $\lambda_{0}^{2}$ in $g$ and then calculate the minimum eigenvalue of $\lambda_{1}^{2}$ in Eq. (5.7), and substitute $\lambda_{1}^{2}$ into Eq. (5.7) again to calculate the minimum eigenvalue $\lambda_{2}^{2}$. Using this iteration process, we can get $\lambda^{2}$ as $\lambda_{i+1}^{2} \approx \lambda_{i}^{2}$. Next, from the relation between $T_{\mathrm{c}}$ and $\lambda^{2}$ we find

$T_{\mathrm{c}}=\frac{1}{4 \pi}\left[(d-1)-\kappa^{2} \lambda^{2} \frac{(d-3)^{2}}{d-2}\right]\left(\frac{\rho}{\lambda}\right)^{\frac{1}{d-2}}$.

In Table 2, the numerical results are exact like [36,37]. We compare the results of the iteration method and the results in [36], and we find that the values from the iteration method are better.

In Sect. 6, we will use the iteration method to analytically research the holographic superconductor in a rotating black string spacetime. 


\section{Analytical investigation of holographic superconductors with backreactions}

The metric of spacetime and the electromagnetical potential of rotating ADS black strings could be given by

$$
\begin{aligned}
\mathrm{d} s^{2}= & -f(r)\left[N(r) \mathrm{d} t-\frac{\omega}{\alpha^{2}} \mathrm{~d} \varphi\right]^{2}+\frac{\mathrm{d} r^{2}}{f(r)} \\
& +r^{2}[N(r) \mathrm{d} \varphi-\omega \mathrm{d} t]^{2}+\alpha^{2} r^{2} \mathrm{~d} z^{2}, \\
\mathrm{~d} A= & N(r) \phi(r) \mathrm{d} t-\frac{\omega}{\alpha^{2}} \phi(r) \mathrm{d} \varphi,
\end{aligned}
$$

where $\omega$ is a constant which is determined by the rotation parameter, and $\Lambda=-3 / \alpha^{2}$ is the cosmological constant. Without loss of generality, we set $\alpha=1$. According to the calculation method in Sect. 2, the Hawking temperature of a black hole (6.1) is [30-34]

$T_{h}=\frac{f^{\prime}\left(r_{h}\right)}{4 \hat{\varrho} \pi}$,

where $\hat{\varrho}=\frac{N}{N^{2}-\omega^{2}}$. Now, let us substitute the metric and electromagnetical potential in Eq. (6.1) into the holographic superconductor action (5.1), and from the variation of the action (5.1), we get four field equations:

$$
\begin{aligned}
& \kappa^{2}\left[\frac{2 \omega^{2} \phi^{2} f^{\prime} N^{\prime}}{r^{2} \omega^{2} N-r^{2} N^{3}}+\frac{2 \phi(r)^{2} N^{\prime \prime}\left(r^{2} N^{2}-\omega^{2} f\right)}{r^{2}\left(N^{3}-\omega^{2} N\right)}\right. \\
& +N^{\prime}\left(\frac{4 \phi \phi^{\prime}\left(r^{2} N^{2}-\omega^{2} f\right)}{r^{2}\left(N^{3}-\omega^{2} N\right)}-\frac{4 N \phi^{2}}{r \omega^{2}-r N^{2}}\right) \\
& +\frac{2 \omega^{2}\left(f-r^{2}\right) \phi^{2} N^{\prime 2}}{r^{2}\left(\omega^{2}-N^{2}\right)^{2}}+4 f \psi^{\prime 2}-\frac{4 \psi^{2} \phi^{2}}{f}+4 m^{2} \psi^{2} \\
& \left.+\phi\left(2 \phi^{\prime \prime}+\frac{4 \phi^{\prime}}{r}\right)\right]+f^{\prime \prime}+\frac{4}{r} f^{\prime}+\frac{2 f}{r^{2}}-12 \\
& +\frac{N^{\prime \prime}\left(-2 r^{2} f\left(\omega^{2}-2 N^{2}\right)+\omega^{2} f^{2}+r^{4} \omega^{2}\right)}{r^{2}\left(N^{3}-\omega^{2} N\right)} \\
& +\frac{\omega^{2}\left(f+r^{2}\right)^{2} N^{\prime 2}}{r^{2}\left(\omega^{2}-N^{2}\right)^{2}}-\frac{N^{\prime}\left(8 f N^{2}-4 \omega^{2} f+4 r^{2} \omega^{2}\right)}{r \omega^{2} N-r N^{3}} \\
& +\frac{N^{\prime}\left[2 \omega^{2} f-2 r^{2}\left(\omega^{2}-2 N^{2}\right)\right]}{r^{2}\left(N^{3}-\omega^{2} N\right)} f^{\prime}=0 \\
& +\phi\left(\frac{N N^{\prime \prime}}{N^{2}-\omega^{2}}-\frac{2 N N^{\prime}}{r \omega^{2}-r N^{2}}-\frac{2 \psi^{2}}{f}\right)=0 \\
& +\frac{N^{\prime 2}\left(\omega^{2} f+r^{2} N^{2}-\kappa^{2} \omega^{2} \phi^{2}\right)}{r^{2} \omega^{2} N-r^{2} N^{3}} \\
& \phi^{\prime \prime}+\phi^{\prime}\left(\frac{2 N N^{\prime}}{N^{2}-\omega^{2}}+\frac{2}{r}\right)-\frac{\omega^{2}\left(r^{2}-f\right) N^{\prime 2}}{r^{2}\left(\omega^{2}-\omega^{2}\right)\left(f^{2} \psi^{\prime 2}+\psi^{2} \phi^{2}\right)} \\
& +\kappa^{2}=0
\end{aligned}
$$

$$
\begin{aligned}
& \psi^{\prime \prime}(r)+\frac{\psi\left(\phi^{2}-m^{2} f\right)}{f^{2}} \\
& +\psi^{\prime}\left(\frac{f^{\prime}}{f}+\frac{2 N N^{\prime}}{N^{2}-\omega^{2}}+\frac{2}{r}\right)=0 .
\end{aligned}
$$

The field $\phi$ must remain finite at event horizon, while as $r \rightarrow \infty$, the boundary condition requires

$$
\begin{aligned}
\psi & =\frac{\sqrt{2}\left\langle\mathcal{O}_{-}\right\rangle}{r^{\Delta_{-}}}+\frac{\sqrt{2}\left\langle\mathcal{O}_{+}\right\rangle}{r^{\Delta_{+}}}+\cdots, \\
\phi & =\mu-\frac{\rho}{r}+\cdots, \\
f & =r^{2}+\cdots, \\
N & =N_{0}+\cdots,
\end{aligned}
$$

where $\rho$ and $\mu$ are, respectively, the charge density and the chemical potential in the dual field theory and $\Delta_{ \pm}=\frac{3}{2} \pm$ $\sqrt{\frac{9}{4}+m^{2}}$, implying that $\Delta$ satisfies $\frac{1}{2}<\Delta<3$. Please note that $N_{0}=1+\omega^{2}$ according to the rotating black string solution given in $[25,26]$.

An effective analytic method proposed by Siopsis and Therrien [35] can be used to investigate the holographic superconductor at the critical temperature. According to the procedure, we should change the radial coordinates as $z=r_{h} / r$, so that the field equations can be rewritten as

$$
\begin{aligned}
& \kappa^{2}\left\{\frac{2 \omega^{2} z^{2} \phi^{2} f_{, z} N_{, z}}{\omega^{2} r_{h}^{2} N-r_{h}^{2} N^{3}}+\frac{2 \phi^{2} N_{, z z}\left(r_{h}^{2} N^{2}-\omega^{2} z^{2} f\right)}{r_{h}^{2}\left(N^{3}-\omega^{2} N\right)}\right. \\
& +N_{, z}\left[\frac{4 \phi \phi, z}{r_{h}^{2}\left(N^{3} N^{2}-\omega^{2} z^{2} f\right)}-\frac{4 \omega^{2} z f \phi^{2}}{r_{h}^{2}\left(N^{3}-\omega^{2} N\right)}\right] \\
& +\frac{2 \omega^{2} \phi^{2} N_{, z}^{2}\left(r_{h}^{2}-z^{2} f\right)}{r_{h}^{2}\left(\omega^{2}-N^{2}\right)^{2}}-\frac{4 r_{h}^{2} \psi^{2} \phi^{2}}{z^{4} f}+4 f \psi_{, z}^{2} \\
& + \\
& \left.+\frac{4 m^{2} r_{h}^{2} \psi^{2}}{z^{4}}+2 \phi \phi_{, z z}\right\}+f_{, z z} \\
& +f_{, z}\left[\frac{N_{, z}\left(2 r_{h}^{2}\left(\omega^{2}-2 N^{2}\right)-2 \omega^{2} z^{2} f\right)}{r_{h}^{2} N\left(\omega^{2}-N^{2}\right)}-\frac{2}{z}\right] \\
& +\frac{z^{2} N_{, z z}\left(-\frac{2 f r_{h}^{2}\left(\omega^{2}-2 N^{2}\right)}{z^{2}}+\omega^{2} f^{2}+\frac{\omega^{2} r_{h}^{4}}{z^{4}}\right)}{r_{h}^{2}\left(N^{3}-\omega^{2} N\right)} \\
& +\frac{\omega^{2} N_{, z}^{2}\left(z^{2} f+r_{h}^{2}\right)^{2}}{z^{2} r_{h}^{2}\left(\omega^{2}-N^{2}\right)^{2}}-\frac{2 \omega^{2} N_{, z}\left(r_{h}^{4}-z^{4} f^{2}\right)}{z^{3} r_{h}^{2}\left(N^{3}-\omega^{2} N\right)} \\
& +\frac{2\left(z^{2} f-6 r_{h}^{2}\right)}{z^{4}}=0,
\end{aligned}
$$

$$
\begin{gathered}
N_{, z z}+\frac{2 N_{, z}}{z}+\frac{N_{, z}^{2}\left(\omega^{2} z^{2} f+r_{h}^{2} N^{2}-\kappa^{2} \omega^{2}-z^{2} \phi^{2}\right)}{\omega^{2} r_{h}^{2} N-r_{h}^{2} N^{3}} \\
+\frac{2 \kappa^{2}\left(N^{2}-\omega^{2}\right)\left(z^{4} f^{2} \psi_{, z}^{2}+r_{h}^{2} \psi^{2} \phi^{2}\right)}{z^{4} f^{2} N}=0,
\end{gathered}
$$




$$
\begin{aligned}
& \phi\left(-\frac{\omega^{2} N_{, z}^{2}\left(r_{h}^{2}-z^{2} f\right)}{r_{h}^{2}\left(\omega^{2}-N^{2}\right)^{2}}-\frac{2 r_{h}^{2} \psi^{2}}{z^{4} f}+\frac{N N_{, z z}}{N^{2}-\omega^{2}}\right) \\
& \quad+\frac{2 N N_{, z} \phi_{, z}}{N^{2}-\omega^{2}}+\phi_{, z z}=0 \\
& \psi_{, z}\left(\frac{f_{, z}}{f}+\frac{2 N N_{, z}}{N^{2}-\omega^{2}}\right)+\frac{r_{h}^{2} \psi\left(\phi^{2}-m^{2} f\right)}{z^{4} f^{2}}+\psi_{, z z}=0 .
\end{aligned}
$$

It is very difficulty to calculate the exact solution from the above equations, but considering the fact that the effect from $\psi$ is very small, at critical temperature we can expand $f, N$, $\phi, \psi$, and the chemical potential $\mu$ as

$$
\begin{aligned}
& \psi=\epsilon \psi_{1}+\epsilon^{3} \psi_{3}+\epsilon^{5} \psi_{5}+\cdots, \\
& \phi=\phi_{0}+\epsilon^{2} \phi_{2}+\epsilon^{4} \phi_{4}+\cdots, \\
& f=f_{0}+\epsilon^{2} f_{2}+\epsilon^{4} f_{4}+\cdots, \\
& N=N_{0}+\epsilon^{2} N_{2}+\epsilon^{4} N_{4}+\cdots, \\
& \mu=\mu_{0}+\epsilon^{2} \mu_{2}+\epsilon^{4} \mu_{4}+\cdots,
\end{aligned}
$$

where $\epsilon=\left\langle\mathcal{O}_{ \pm}\right\rangle \ll 1$. Thus, the calculation accuracy is enough to consider the first terms in Eq. (6.12).

Near the critical temperature point, the scalar field $\psi$ vanishes and we can use $N_{0}$ to replace $N$, so that Eq. (6.10) has the solution

$$
A_{t} \approx N_{0} \phi_{0}=\lambda r_{h c}(1-z),
$$

where $\lambda=\rho r_{h c}^{-2}$, and the $r_{h c}$ is the position of the event horizon at the critical temperature point. For convenience, we set $\hat{\lambda}=\frac{\lambda}{N_{0}}$ and $\hat{\rho}=\frac{\rho}{N_{0}}$. Next, substituting Eq. (6.13) into Eq. (6.8), we obtain

$$
f_{0}=r_{h c}^{2}\left[z^{-2}-z+\kappa^{2} \hat{\lambda}^{2} \frac{z}{2}(z-1)\right] \text {. }
$$

Let us set $\psi_{1} \sim \frac{\left\langle\mathcal{O}_{ \pm}\right\rangle}{r_{h c}^{\Delta}} z^{\Delta} F_{0}(z)$, so that Eq. (6.11) becomes

$$
\begin{gathered}
F_{0, z z}+\left(\frac{f_{0, z}}{f_{0}}+\frac{2 N_{0} N_{0, z}}{N_{0}^{2}-\omega^{2}}+\frac{2 \Delta}{z}\right) F_{0, z} \\
+\left[\frac{\Delta f_{0, z}}{z f_{0}}-\frac{m^{2} r_{h c}^{2}}{z^{4} f}+\frac{\hat{\lambda}^{2}(z-1)^{2} r_{h c}^{4}}{z^{4} f_{0}^{2}}\right. \\
\left.\quad-\frac{2 \Delta N_{0} N_{0, z}}{\omega^{2} z-z N_{0}^{2}}+\frac{(\Delta-1) \Delta}{z^{2}}\right] F_{0}=0 .
\end{gathered}
$$

Therefore, from the Sturm-Liouville eigenvalue problem, we get, as in (5.7),

$\lambda^{2}=\frac{\int_{0}^{1} T_{0}\left(F_{0}^{\prime 2}-U_{0} F_{0}^{2}\right) \mathrm{d} z}{\int_{0}^{1} T_{0} V_{0} F_{0}^{2} \mathrm{~d} z}$, where

$$
\begin{aligned}
T_{0} & =r_{h c^{2}}^{2} z^{2 \Delta}\left(\frac{1}{z^{2}}+\frac{1}{2} \kappa^{2} \hat{\lambda}^{2}(z-1) z-z\right), \\
U_{0} & =\frac{4}{\left(\kappa^{2} \hat{\lambda}^{2} z^{3}-2\left(z^{2}+z+1\right)\right)^{2}}, \\
V_{0} & =\frac{\Delta \kappa^{2} \hat{\lambda}^{2} z^{3}(-\Delta+\Delta z+z)-2 m^{2}}{\kappa^{2} \hat{\lambda}^{2} z^{6}-z^{5}\left(\kappa^{2} \hat{\lambda}^{2}+2\right)+2 z^{2}} \\
& -\frac{2 \Delta\left(\Delta\left(z^{3}-1\right)+3\right)}{\kappa^{2} \hat{\lambda}^{2} z^{6}-z^{5}\left(\kappa^{2} \hat{\lambda}^{2}+2\right)+2 z^{2}},
\end{aligned}
$$

where we assume $F(z)=1-a z^{2}$, so that we can use the iteration method to calculate the solution for $\lambda$. Finally, we can compute the temperature (6.2) by

$T_{\mathrm{c}}=\frac{f_{0}^{\prime}\left(r_{h c}\right)}{4 \varrho_{0} \pi}=\frac{6-\kappa^{2} \hat{\lambda}^{2}}{8 \varrho_{0} \pi} r_{h c}=\frac{6-\kappa^{2} \hat{\lambda}^{2}}{8 \pi \varrho_{0}} \sqrt{\frac{\rho}{\hat{\lambda}}}$,

where $\varrho_{0}=\frac{N_{0}}{N_{0}^{2}-\omega^{2}}$. Comparing with the critical temperature of statical black strings in [36], we found that the whole correction of the rotation parameter $\omega$ comes from $\varrho_{0}$, while the process of calculating $\hat{\lambda}$ by Eq. (6.15) does not depend on $\omega$.

Now, we compare the analytical solution with the exact numerical solution in Table 3 . Because the calculation of $\hat{\lambda}$ does not depend on the rotation parameter, while the correction of $\omega$ is included in $\varrho_{0}$, we can set $\varrho_{0}=1$ without loss of generality.

On the other hand, we also calculate the $T_{\mathrm{c}}$ with different $\kappa$ and $\Delta$ in Table 4.

The results show that the critical temperature $T_{\mathrm{c}}$ will decrease as $\kappa$ or $\Delta$ increase, and from the relationship $\hat{\eta}=\frac{\sqrt{\rho}}{\varrho_{0}}$, we also find that the effect of the rotation parameter $\omega$ also is a decrease of $T_{\mathrm{c}}$.

Table 3 Comparing the $T_{\mathrm{c}}$ results of the iteration method and the numerical exact solution $[36,37]$ in the 4-dimensional AdS black hole background as $m^{2}=-2$

\begin{tabular}{llllll}
\hline$\kappa$ & $\Delta=1$ & & & $\Delta=2$ & \\
\cline { 2 - 3 } & Analytical & Numerical & & Analytical & Numerical \\
\hline 0 & $0.2250 \rho^{1 / 2}$ & $0.2255 \rho^{1 / 2}$ & & $0.1170 \rho^{1 / 2}$ & $0.1184 \rho^{1 / 2}$ \\
0.05 & $0.2249 \rho^{1 / 2}$ & $0.2253 \rho^{1 / 2}$ & & $0.1163 \rho^{1 / 2}$ & $0.1177 \rho^{1 / 2}$ \\
0.10 & $0.2246 \rho^{1 / 2}$ & $0.2250 \rho^{1 / 2}$ & & $0.1142 \rho^{1 / 2}$ & $0.1156 \rho^{1 / 2}$ \\
0.15 & $0.2241 \rho^{1 / 2}$ & $0.2245 \rho^{1 / 2}$ & & $0.1107 \rho^{1 / 2}$ & $0.1121 \rho^{1 / 2}$ \\
0.20 & $0.2235 \rho^{1 / 2}$ & $0.2239 \rho^{1 / 2}$ & & $0.1060 \rho^{1 / 2}$ & $0.1074 \rho^{1 / 2}$ \\
0.25 & $0.2226 \rho^{1 / 2}$ & $0.2230 \rho^{1 / 2}$ & & $0.1003 \rho^{1 / 2}$ & $0.1017 \rho^{1 / 2}$ \\
0.30 & $0.2216 \rho^{1 / 2}$ & $0.2220 \rho^{1 / 2}$ & & $0.0938 \rho^{1 / 2}$ & $0.0951 \rho^{1 / 2}$ \\
& & & & &
\end{tabular}


Table 4 Comparing the $T_{\mathrm{c}}$ results of the iteration method and the numerical exact solution in the 4-dimensional AdS black hole background, where $\hat{\eta}=\frac{\sqrt{\rho}}{\varrho_{0}}$

\begin{tabular}{lllll}
\hline$\Delta$ & $\kappa=0$ & $\kappa=0.1$ & $\kappa=0.2$ & $\kappa=0.3$ \\
\hline 0.6 & $0.4550 \hat{\eta}$ & $0.4550 \hat{\eta}$ & $0.4549 \hat{\eta}$ & $0.4547 \hat{\eta}$ \\
0.8 & $0.2912 \hat{\eta}$ & $0.2911 \hat{\eta}$ & $0.2906 \hat{\eta}$ & $0.2898 \hat{\eta}$ \\
1 & $0.2250 \hat{\eta}$ & $0.2246 \hat{\eta}$ & $0.2235 \hat{\eta}$ & $0.2216 \hat{\eta}$ \\
1.2 & $0.1864 \hat{\eta}$ & $0.1857 \hat{\eta}$ & $0.1837 \hat{\eta}$ & $0.1804 \hat{\eta}$ \\
1.4 & $0.1607 \hat{\eta}$ & $0.1596 \hat{\eta}$ & $0.1564 \hat{\eta}$ & $0.1513 \hat{\eta}$ \\
1.6 & $0.1421 \hat{\eta}$ & $0.1406 \hat{\eta}$ & $0.1359 \hat{\eta}$ & $0.1286 \hat{\eta}$ \\
1.8 & $0.1281 \hat{\eta}$ & $0.1259 \hat{\eta}$ & $0.1196 \hat{\eta}$ & $0.1099 \hat{\eta}$ \\
2 & $0.1170 \hat{\eta}$ & $0.1142 \hat{\eta}$ & $0.1060 \hat{\eta}$ & $0.0938 \hat{\eta}$ \\
2.2 & $0.1081 \hat{\eta}$ & $0.1045 \hat{\eta}$ & $0.0943 \hat{\eta}$ & $0.0795 \hat{\eta}$ \\
2.4 & $0.1007 \hat{\eta}$ & $0.0962 \hat{\eta}$ & $0.0839 \hat{\eta}$ & $0.0666 \hat{\eta}$ \\
2.6 & $0.0944 \hat{\eta}$ & $0.0890 \hat{\eta}$ & $0.0745 \hat{\eta}$ & $0.0550 \hat{\eta}$ \\
2.8 & $0.0890 \hat{\eta}$ & $0.0827 \hat{\eta}$ & $0.0660 \hat{\eta}$ & $0.0446 \hat{\eta}$ \\
\hline
\end{tabular}

\section{Conclusions}

We have considered holographic superconductors in 3+1dimensional rotating black strings. The investigation shows that the $A_{\varphi}=\Omega$ term can be ignored in 3+1-dimensional static spacetime. The effect from the rotation parameter $a$ leads the real part of the conductivity to be smaller as the rotation increases. On the other hand, the frequency times the imaginary part increases in absolute value. Superconductivity, however, remains. This may imply that the presence of spin can eventually minimize superconductivity in a physical system.

On the other hand, we proposed an iteration method to improve the analytical calculation for holographic superconductors with backreactions, and it is proved that we can use this method to get better results. We also use this method to research the holographic superconductor in a rotating spacetime, while the results point out that the influence of the rotation parameter mainly concentrates on $\varrho_{0}$ terms, which could make the critical temperature decrease. At the same time, the backreactions also could lead $T_{\mathrm{c}}$ to decrease.

The spacetime we considered in this paper is the spacetime of rotating black strings, while it could be more meaningful to study further rotating black hole case. Some work as regards the superconducting instability of the Kerr-Newmann-de Sitter black hole appeared in [38]. Further work on the holographic superconductor in Kerr-Newmann-de Sitter spacetime is under way. On the other hand, recently Hořava proposed a new gravity theory, Hořava-Lifshitz gravity [39-46], which could solve several difficulties in quantum gravity and cosmology such as dark energy and dark matter, and our works [47-49] have proved this theory to satisfy the results of post-Newtonian approximations, so it will be very interest- ing to research the holographic superconductors in HořavaLifshitz gravity with backreaction. Further study is under way.

Acknowledgments This work was supported in part by FAPESP No. 2012/08934-0 and CNPq.

Open Access This article is distributed under the terms of the Creative Commons Attribution License which permits any use, distribution, and reproduction in any medium, provided the original author(s) and the source are credited.

Funded by $\mathrm{SCOAP}^{3}$ / License Version CC BY 4.0.

\section{References}

1. S.A. Hartnoll, C.P. Herzog, G.T. Horowitz, Phys. Rev. Lett. 101, 031601 (2008)

2. S.A. Hartnoll, C.P. Herzog, G.T. Horowitz, J. High Energy Phys. 12, 015 (2008)

3. C.P. Herzog, J. Phys. A 42, 343001 (2009)

4. S.A. Hartnoll, Class. Quantum Grav. 26, 224002 (2009)

5. G.T. Horowitz, Lect. Notes Phys. 828, 313 (2011). arXiv:1002.1722 [hep-th]

6. G.T. Horowitz, R.C. Myers, Phys. Rev. D 59, 026005 (1999)

7. T. Nishioka, S. Ryu, T. Takayanagi, J. High Energy Phys. 03, 131 (2010)

8. G.T. Horowitz, B. Way, J. High Energy Phys. 11, 011 (2010)

9. Y. Peng, Q.Y. Pan, B. Wang, Phys. Lett. B 699, 383 (2011)

10. P. Basu, F. Nogueira, M. Rozali, J.B. Stang, M.V. Raamsdonk, New J. Phys. 13, 055001 (2011)

11. Y. Brihaye, B. Hartmann, Phys. Rev. D 83, 126008 (2011)

12. R.G. Cai, S. He, L. Li, Y.L. Zhang, J. High Energy Phys. 07, 088 (2012)

13. Y. Peng, X.M. Kuang, Y.Q. Liu, B. Wang, arXiv:1204.2853 [hepth]

14. Y.Q. Wang, Y.X. Liu, R.G. Cai, S. Takeuchi, H.Q. Zhang, J. High Energy Phys. 09, 058 (2012)

15. A. Akhavan, M. Alishahiha, Phys. Rev. D 83, 086003 (2011). arXiv: 1011.6158 [hep-th]

16. R.G. Cai, H.F. Li, H.Q. Zhang, Phys. Rev. D 83, 126007 (2011). arXiv:1103.5568 [hep-th]

17. Q.Y. Pan, J.L. Jing, B. Wang, Phys. Rev. D 84, 126020 (2011)

18. Chong Oh Lee, Eur. Phys. J. C 72, 2092 (2012)

19. R.G. Cai, X. He, H.F. Li, H.Q. Zhang, Phys. Rev. D 84, 046001 (2011)

20. R.G. Cai, L. Li, H.Q. Zhang, Y.L. Zhang, Phys. Rev. D 84, 126008 (2011)

21. J.P. Wu, Y. Cao, X.M. Kuang, W.J. Li, Phys. Lett. B 697, 153 (2011)

22. E. Abdalla, et al.,Phys Lett. B 709, 276-279(2012)

23. E. Abdalla, et al., Phys. Rev. D 82, 124033 (2010)

24. J.P.S. Lemos Phys. Lett. B 353, 46 (1995). gr-qc/9404041

25. S. Salarpour, A. Sheykhi, Y. Bahrampour, Phys. Scr. 87, 045004 (2013). arXiv:1304.3057

26. A. Sheykhi, Phys. Rev. D 78, 064055 (2008)

27. S.P. Robinson, F. Wilczek, Phys. Rev. Lett. 95, 011303 (2005). arXiv:gr-qc/0502074

28. P. Kraus, F. Wilczek, Nucl. Phys. B 433, 403 (1995). arXiv:gr-qc/9408003

29. J.Y. Zhang, Z. Zhao, Phys. Lett. B 638, 110 (2006). arXiv:gr-qc/0512153

30. K. Srinivasan, T. Padmanabhan, Phys. Rev. D 60, 24007 (1999)

31. R. Kerner, R.B. Mann, Class. Quantum Grav. 25, 095014 (2008). arXiv:0710.0612 [hep-th] 
32. K. Lin, S.Z. Yang, Phys. Rev. D 79, 064035 (2009)

33. K. Lin, S.Z. Yang, Phys. Lett. B 674, 127 (2009)

34. K. Lin, S.Z. Yang, Chin. Phys. B 11, 110403 (2011)

35. G. Siopsis, J. Therrien, J. High Energy Phys. 05, 013 (2010). arXiv: 1003.4275

36. Q.Y. Pan, J.L. Jing, B. Wang, S.B. Chen, J. High Energy Phys. 06, 087 (2012). arXiv: 1205.3543

37. G.T. Horowitz, M.M. Roberts, Phys. Rev. D 78, 126008 (2008). arXiv:0810.1077

38. J. Sonner, Phys. Rev. D 80, 084031 (2009). arXiv:0903.0627

39. P. Hořava, Phys. Rev. D 79, 084008 (2009). arXiv:0901.3775

40. A. Wang, Phys. Rev. D 82, 124063 (2010). arXiv:1008.3637

41. A. Borzou, K. Lin, A. Wang, J. Cosmol. Astropart. Phys. 05, 006 (2011). arXiv:1103.4366
42. S. Mukohyama, J. Cosmol. Astropart. Phys. 06, 001 (2009). arXiv:0904.2190

43. T. Zhu, Q. Wu, A. Wang, F.-W. Shu, Phys. Rev. D 84, 101502 (R) (2011) arXiv:1108.1237

44. T. Zhu, F.-W. Shu, Q. Wu, A. Wang, Phys. Rev. D 85, 044053 (2012). arXiv:1110.5106

45. A. Wang, Y. Wu, Phys. Rev. D 83, 044031 (2011). arXiv: 1009.2089

46. K. Lin, A. Wang, Q. Wu, T. Zhu, Phys. Rev. D 84, 044051 (2011). arXiv: 1106.1486

47. K. Lin, S. Mukohyama, A. Wang, Phys. Rev. D 86, 104024 (2012). arXiv: 1206.1338

48. K. Lin, A. Wang, Phys. Rev. D 87, 084041 (2013). arXiv: 1212.6794

49. K. Lin, S. Mukohyama, A. Wang, T. Zhu, Phys. Rev. D 89, 084022 (2014). arXiv:1310.6666 